\title{
Parallelized Hashing via $j$-Lanes and $j$-Pointers Tree Modes, with Applications to SHA-256
}

\author{
Shay Gueron ${ }^{1,2}$ \\ ${ }^{1}$ Department of Mathematics, University of Haifa, Haifa, Israel \\ ${ }^{2}$ Intel Corporation, Israel Development Center, Haifa, Israel \\ Email: shay@math.haifa.ac.il
}

Received 1 May 2014; revised 1 June 2014; accepted 28 June 2014

Copyright (C 2014 by author and Scientific Research Publishing Inc.

This work is licensed under the Creative Commons Attribution International License (CC BY). http://creativecommons.org/licenses/by/4.0/

c) (i) Open Access

\begin{abstract}
$\boldsymbol{j}$-lanes tree hashing is a tree mode that splits an input message into $\boldsymbol{j}$ slices, computes $\boldsymbol{j}$ independent digests of each slice, and outputs the hash value of their concatenation. $j$-pointers tree hashing is a similar tree mode that receives, as input, $j$ pointers to $j$ messages (or slices of a single message), computes their digests and outputs the hash value of their concatenation. Such modes expose parallelization opportunities in a hashing process that is otherwise serial by nature. As a result, they have a performance advantage on modern processor architectures. This paper provides precise specifications for these hashing modes, proposes appropriate IVs, and demonstrates their performance on the latest processors. Our hope is that it would be useful for standardization of these modes.
\end{abstract}

\section{Keywords}

Tree Mode Hashing, SHA-256, SIMD Architecture, Advanced Vector Extensions Architectures, AVX, AVX2

\section{Introduction}

This paper expands upon the $j$-lanes tree hashing mode which was proposed in [1]. It provides specifications, enhancements, and an updated performance analysis. The purpose is to suggest such modes for standardization. Although the specification is general, we focus on $j$-lanes tree hashing with SHA-256 [2] as the underlying hash function.

The $j$-lanes mode is a particular form of tree hashing, which is optimized for contemporary architectures of 
modern processors that have SIMD (Single Instruction Multiple Data) instructions. Currently deployed SIMD architectures use either 128-bit (e.g., SSE, AVX [3], NEON [4]) or 256-bit (AVX2 [3]) registers. For SHA-256, an algorithm that (by its definition) operates on 32-bit words, AVX and AVX2 architectures can process 4 or 8 "lanes" in parallel, respectively. The $j$-lanes mode capitalizes on this parallelization capability.

The AVX2 architecture [3] includes all the necessary instructions to implement SHA-256 operations efficiently: 32-bit shift (vpsrld) and add (vpaddd), bitwise logical operations (vpandn, vpand, vpxor), and the 32-bit rotation (by combining two shifts (vpsrld/vpslld) with a single xor/or (vpxor) operation).

The future AVX512f instructions set [3] [5] supports 512-bit registers, ready for operating on 16 lanes. It also adds a few useful instructions that would increase the parallelized hashing performance: rotation (vprold) and ternary-logic operation (vpternlogd). The (vpternlogd) instruction allows software to use a single instruction for implementing logical functions such as Majority and Choose, which SHA-256 (and other hash algorithms) use. Rotation (vprold) can perform the SHA-256 rotations faster than the vpsrld + vpslld + vpxor combination.

\section{Preliminaries}

Hereafter, we focus on hash functions (HASH) that use the Merkle-Damgård construction (SHA-256, SHA-512, SHA-1 are particular examples). Other constructions can be handled similarly. Suppose that HASH produces a digest of $d$ bits, from an input message $M$ whose length is length $(M)$. The hashing process starts from an initial state, of size $i$ bits, called an Initialization Vector (denoted HashIV). The message is first padded with a fixed string plus the encoded length of the message. The resulting (padded) message is then viewed and processed as the concatenation $M||$ padding $=m_{0}\left\|m_{1}\right\| \ldots \| m_{k-1}$ of $k$ consecutive fixed size blocks $m_{0} m_{1} \ldots m_{k-1}$.

The output digest is computed by an iterative invocation of a compression function compress ( $H, B L O C K)$. The inputs to the compression function are a chaining variable $(H)$ of $i$ bits, and a block (BLOCK) of $b$ bits. Its output is an $i$-bit value that can be used as the input to the next iteration. The output digest (of HASH) is $f\left(H^{k-1}\right)$. We call an invocation of the compression function an "Update" (because it updates the chaining variable).

We use here the following notations:

- $\lceil x\rceil:$ floor $(x)$.

- $[x]: \operatorname{ceil}(x)=$ floor $(x+1)$.

- $S[y: x]$ : bits $x$ through $y$ of $S$.

- $\quad \|$ : string concatenation (e.g., $04 \| 08=0408$ ).

- HASH: the underlying hash function; HASH = HASH (message, length (message)).

- HashIV the Initialization Vector used for HASH (e.g., for SHA-256 Hash IV = 0x6a09e667, 0xbb67ae85, $0 x 3 c 6 e f 372,0 x a 54 f f 53 a, 0 x 510 e 527 f, 0 x 9 b 05688 c, 0 x 1 f 83 d 9 a b, 0 x 5 b e 0 c d 19$; when written as 8 integers).

- compress (H, BLOCK): the compression function used by HASH. It consumes a single fixed sized data chunk (BLOCK) of the message, a state $(H)$, and updates $H$ (at output) according to a specified algorithm ([2] defines the compression function for SHA-256).

- $M$ : the hashed message.

- $N$ : the length, in bits, of $M$.

- $L$ : the length, in bytes, of $M(L=[N / 8])$.

- $d$ : the length, in bits, of the digest that $H A S H$ produces.

- $D$ : the length, in bytes, of the digest that $H A S H$ produces $(D=[d / 8])$.

- $B$ : the length, in bytes, of the message block consumed by the compression function compress (e.g., for SHA-256, $B=64$ ).

- $j$ : the number of lanes used by the $j$-lanes hashing process (in this paper, we discuss only $j=4,8,16$ ).

- $Q$ : the size, in bits, of the "word" that HASH uses during the computations $(Q=32$ for SHA-256, and $Q=64$ for SHA-512).

- $W$ : the size, in bytes, of the "word" that $H A S H$ uses during the computations $(W=Q / 8)$.

- $S$ : the number of lanes that a given architecture supports, with respect to the word size of HASH (e.g., AVX architecture has registers (xmm's) that can hold 128 bits. For HASH = SHA-256, $Q=32$, therefore, $S=128 / Q$ $=4)$.

- $P$ : the length, in bytes, of the minimal padding length of HASH (for SHA-256, a bit "1" is concatenated, and then the message bit length $(N)$, encoded as an 8-byte Big Endian integer. Therefore, with SHA-256, we have $P=9$ ). 


\section{The $j$-Lanes Tree Hash}

The $j$-lanes tree hash is defined in the context of the underlying hash function HASH, and $j(j \geq 2)$ is a parameter. We are interested here in $j=4,8,16$. The input to the $j$-lanes hash function is a message $M$ whose length is $N$ bits.

This message is (logically) divided into $k(k \geq 0)$ consecutive $Q$-bit "words" $m_{i}, i=0,1, \ldots, k-1$ (if $M$ is the NULL message, then $k=0)$.

When $k \geq 1$, the words $m_{j}, j=0,1, \ldots, k-2$ (if $k-2<0$, there are no words in the count) consist of $Q$ bits each. If $N$ is not divisible by $Q$, then the last word $m_{k-1}$ is incomplete, and consists of only $(N \bmod Q)$ bits.

We then split the original message $M$ into the $j$ disjoint sub-messages (buffers) Buff, Buff $1, \ldots$, Buff $f_{-1}$ as follows:

Buff $_{0}=\mathrm{m}_{0}\left\|\mathrm{~m}_{j}\right\| \mathrm{m}_{j \times 2} \ldots$

Buff $_{1}=\mathrm{m}_{1}\left\|\mathrm{~m}_{j+1}\right\| \mathrm{m}_{j \times 2+1} \ldots$

...

$\operatorname{Buff}_{j-1}=\mathrm{m}_{j-1}\left\|\mathrm{~m}_{j \times 2-1}\right\| \mathrm{m}_{j \times 3-1} \ldots$

Note if $N \leq Q \times(j-1)$, then one or more buffers Buff $i$ will be a $N U L L$ buffer. If $N=0$ all the buffers are defined to be $N U L L$, and will be hashed as the empty message (i.e. only the padding pattern is hashed in that case).

After the message is split into $j$ disjoint buffers, as described above, the underlying hash function, HASH, is independently applied to each buffer as follows:

$\mathrm{H}_{0}=$ HASH $\left(\right.$ Buff $_{0}$, length $\left(\right.$ Buff $\left.\left._{0}\right)\right)$

$\mathrm{H}_{1}=\mathrm{HASH}\left(\right.$ Buff $_{1}$, length $\left(\right.$ Buff $\left.\left._{1}\right)\right)$

$\mathrm{H}_{2}=\mathrm{HASH}\left(\right.$ Buff $_{2}$, length $\left(\right.$ Buff $\left.\left._{2}\right)\right)$

...

$\mathrm{H}_{j-1}=$ HASH $\left(\right.$ Buff $_{j-1}$, length $\left(\right.$ Buff $\left.\left._{j-1}\right)\right)$

The $j$-lanes digest $(\mathrm{H})$ is defined by

$\mathrm{H}=\operatorname{DIGEST}(\mathrm{HASH}, \mathrm{M}$, length $(\mathrm{M}), j)=\mathrm{HASH}\left(\mathrm{H}_{0}\left\|\mathrm{H}_{1}\right\| \mathrm{H}_{2}\|\ldots\| \mathrm{H}_{j-1}, j \times \mathrm{D}\right)$

Remark 1: The final stage of the process is called the wrapping stage. It hashes a message with a fixed size of $j \times D$ bytes. The number of updates required is $[(j \times D+P) / B\rceil$ that are likely to be serial updates.

Remark 2: The API for a $j$-lanes hash for a fixed $j$ would be the same as for the underlying hash, i.e. for SHA-256, the $j$-lanes implementation could have the following API: SHA256_j_lanes (uint8_t* hash, uint8_t* msg, size_tlen).

Example 1: Consider a message $M$ with $N=4096$ bits, and the hash function HASH = SHA-256 that operates on 32-bit words $(Q=32)$. Here, $k=\lceil 4096 / 32\rceil=128$. For $j=8$ we get

$$
\begin{aligned}
\text { Buff }_{0} & =\mathrm{m}_{0}\left\|\mathrm{~m}_{8}\right\| \mathrm{m}_{16} \ldots \| \mathrm{m}_{120} \\
\text { Buff }_{1} & =\mathrm{m}_{1}\left\|\mathrm{~m}_{9}\right\| \mathrm{m}_{17} \ldots \| \mathrm{m}_{121} \\
\text { Buff }_{2} & =\mathrm{m}_{2}\left\|\mathrm{~m}_{10}\right\| \mathrm{m}_{18} \ldots \| \mathrm{m}_{122} \\
\text { Buff }_{3} & =\mathrm{m}_{3}\left\|\mathrm{~m}_{11}\right\| \mathrm{m}_{19} \ldots \| \mathrm{m}_{123} \\
\text { Buff }_{4} & =\mathrm{m}_{4}\left\|\mathrm{~m}_{12}\right\| \mathrm{m}_{20} \ldots \| \mathrm{m}_{124} \\
\text { Buff }_{5} & =\mathrm{m}_{5}\left\|\mathrm{~m}_{13}\right\| \mathrm{m}_{21} \ldots \| \mathrm{m}_{125} \\
\text { Buff }_{6} & =\mathrm{m}_{6}\left\|\mathrm{~m}_{14}\right\| \mathrm{m}_{22} \ldots \| \mathrm{m}_{126} \\
\text { Buff }_{7} & =\mathrm{m}_{7}\left\|\mathrm{~m}_{15}\right\| \mathrm{m}_{23} \ldots \| \mathrm{m}_{127}
\end{aligned}
$$

where each one of the eight buffers is 512 bit long.

Example 2: Consider a message $M$ with $N=2913$ bits, and HASH $=$ SHA-256 $(Q=32)$. Here, $k=\lceil 2913 / 32\rceil$ $=92$. Since $2913 \bmod 32=1$, the last word, $m_{91}$, consists of only a single bit. For $j=8$, we get

$$
\begin{aligned}
& \text { Buff }_{0}=\mathrm{m}_{0}\left\|\mathrm{~m}_{8}\right\| \mathrm{m}_{16} \ldots\left\|\mathrm{m}_{80}\right\| \mathrm{m}_{88} \\
& \text { Buff }_{1}=\mathrm{m}_{1}\left\|\mathrm{~m}_{9}\right\| \mathrm{m}_{17} \ldots\left\|\mathrm{m}_{81}\right\| \mathrm{m}_{89} \\
& \text { Buff }_{2}=m_{2}\left\|m_{10}\right\| m_{18} \ldots .\left\|m_{82}\right\| m_{90} \\
& \text { Buff }_{3}=m_{3}\left\|m_{11}\right\| m_{19} \ldots\left\|m_{83}\right\| m_{91} \\
& \text { Buff }_{4}=\mathrm{m}_{4}\left\|\mathrm{~m}_{12}\right\| \mathrm{m}_{20} \ldots \| \mathrm{m}_{84} \\
& \text { Buff }_{5}=\mathrm{m}_{5}\left\|\mathrm{~m}_{13}\right\| \mathrm{m}_{21} \ldots \| \mathrm{m}_{85} \\
& \text { Buff }_{6}=\mathrm{m}_{6}\left\|\mathrm{~m}_{14}\right\| \mathrm{m}_{22} \ldots \| \mathrm{m}_{86} \\
& \text { Buff }_{7}=\mathrm{m}_{7}\left\|\mathrm{~m}_{15}\right\| \mathrm{m}_{23} \ldots \| \mathrm{m}_{87}
\end{aligned}
$$

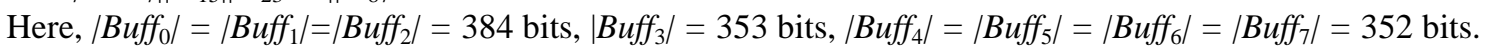


Example 3: Consider a message $M$ with $N=100$ bits, and HASH = SHA-256 $(Q=32)$. Here, $k=\lceil 100 / 32\rceil=$ 4. Since $100 \bmod 32=4$, the last word, $m_{3}$, consists of only 4 bits. For $j=8$, we get

Buff $_{0}=\mathrm{m}_{0}$

Buff $_{1}=\mathrm{m}_{1}$

Buff $_{2}=\mathrm{m}_{2}$

Buff $_{3}=m_{3}$

$\mathrm{Buff}_{4}=$ NULL

$\mathrm{Buff}_{5}=$ NULL

Buff $_{6}=$ NULL

$\mathrm{Buff}_{7}=$ NULL

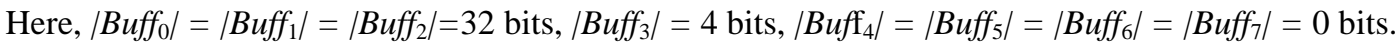

Remark 3: Similarly to the serial hashing, the $j$-lanes hashing can process the message incrementally (e.g., when the messages is streamed). Since the parallelized compression operates (in parallel) on consecutive blocks of $j \times B$ bytes, it needs to receive only the "next $j \times B$ bytes" in order to compute an Update.

\section{The $j$-Pointers Tree Hash}

An alternative way to define $j$ "slices" of the message $M$, is to provide $j$ pointers to $j$ disjoint buffers $B u f f_{0}, \ldots$, Buff $f_{j-1}$, of $M$, together with $k$ values for the length of each buffer. In this case, it is also required that $\Sigma_{i}$ length (Buff $f_{i}$ $=$ length $(M)$.

In this case, the $j$-pointers tree hash procedure would be the following. Compute the $j$ hash values for each of the disjoint buffers:

$\mathrm{H}_{0}=$ HASH (Buff 0 , length (Buff $\left.)_{0}\right)$ )

$\mathrm{H}_{1}=$ HASH $\left(\right.$ Buff $_{1}$, length $\left(\right.$ Buff $\left.\left._{1}\right)\right)$

$\mathrm{H}_{2}=$ HASH $\left(\right.$ Buff $_{2}$, length $\left(\right.$ Buff $\left.\left._{2}\right)\right)$

...

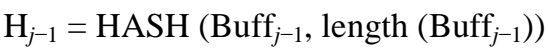

Produce the output digest

$\mathrm{H}=\mathrm{HASH}\left(\mathrm{H}_{0}\left\|\mathrm{H}_{1}\right\| \mathrm{H}_{2}\|\ldots\| \mathrm{H}_{j-1}, j \times \mathrm{D}\right)$

Remark 4: In a software implementation, the API of the $j$-lanes function is the same as the API for any other hash function (see Remark 2).The function computes the buffers and their length internally. On the other hand, the API to a $j$-pointers hash requires a pointer to each buffer and its length, to be provided by the caller. For example:

SHA256_4_pointers(uint8_t* hash, uint8_t* buffo, size_tlen0, uint8_t* buff1, size_tlen1, uint8_t* buff2, size_tlen2, uint8_t* buff3, size_tlen3)

or, alternatively:

SHA256_j_pointers(uint8_t* hash, uint8_t** buffs, size_t*lengths, unsigned int $j$ )

\section{The Difference between $j$-Pointers Tree Hash and $j$-Lanes Tree Hash}

The $j$-pointers and the $j$-lanes tree modes are essentially the same construction, and the difference is in how the message is viewed (logically) as $j$ slices. The $j$-lanes tree mode has a performance advantage when implemented on SIMD architectures because it supports natural sequential loads into the SIMD registers: each word is naturally placed in the correct lane (see Figure 1).

The $j$-pointers tree mode expects the data to be loaded from $j$ locations. It is more suitable for implementations on multi-processor platforms, and for hashing multiple independent messages into a single digest (e.g., hashing a complete file-system while keeping a single digest). Of course, a $j$-pointers tree can also be used on a SIMD architecture, but in that case it requires "transposing" the data in order to place the words in the correct position in the registers. This (small) overhead is saved by using the $j$-lanes tree mode.

\section{Counting the Number of Updates}

The performance of a standard (serial) hash function is closely proportional to the number of Updates $(U)$ that the computations involve, namely 


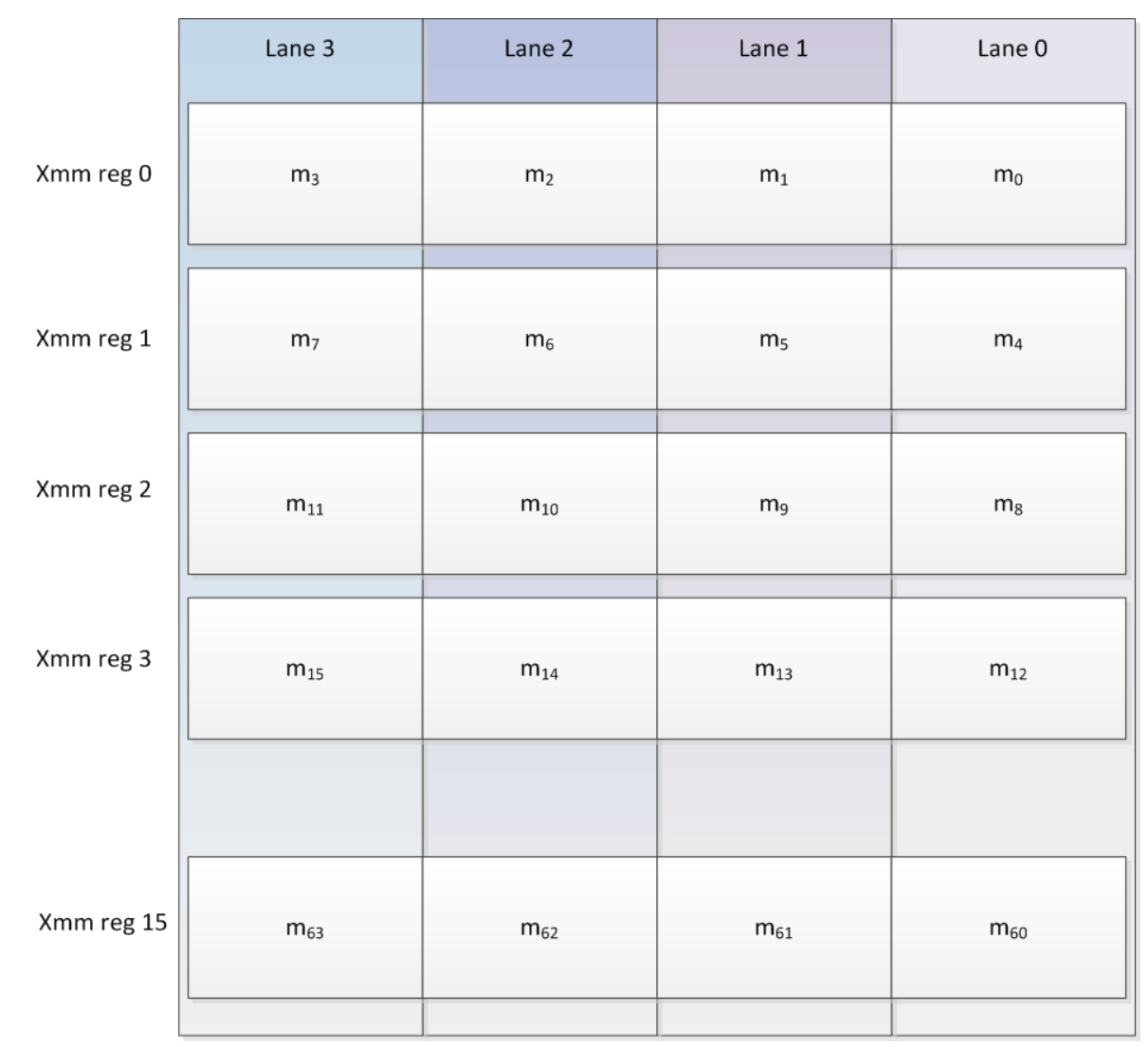

Figure 1. The $j$-lanes tree mode natural data alignment with SIMD architectures (here, with 128-bit registers (xmm'a) as 4 32-bit words).

$$
U=\lceil(L+P) / B\rceil
$$

In Equation (1), each Update consumes $B$ additional bytes of the (padded) message, and the number of bytes in the padded message is at least $L+P$ (with no more than a single block added by the padding).

For the $j$-lanes hash (with the underlying function HASH), the number of serially computed Updates can be approximated by

$$
U \leq\lceil L /(\min (j, S) \times B)\rceil+1+\lceil(j \times D+P) / B\rceil
$$

Note that some of the $j$-lanes Updates are carried out in parallel, compressing $\min (S, j)$ blocks per one Update call. Equation (2) accounts for parallelizing at most $\min (S, j)$ block compressions, thus contributing the term $\lceil L /(\min (j, S) \times B)]$, plus one Update for the padding block. A padding block is counted for each lane, although, depending on the length of the message, some Updates are redundant. The wrapping step cannot be parallelized (in general) and adds $\lceil(j \times D+P) / B\rceil$ serial Updates to the count.

Example 4: Suppose that HASH = SHA-256, and consider a message of 1024 bytes. The standard SHA-256 function requires $[(1024+9) / 64]=17$ Updates. We compare this to the count of $j$-lanes Updates for a few values of $j$ :

For the AVX2 architecture (Haswell architecture [3]) we have $\mathrm{D}=32, B=64, P=9, S=8$. This implies that the 8-lanes SHA-256 $(j=8)$ is optimal. It requires $[1024 /(8 \times 64)\rceil+1+\lceil(8 \times 32+9) / 64\rceil=8$ Updates.

For the AVX architecture (Sandy Bridge architecture), we have $S=4$, so, $j=4$ is the optimal choice for this setup, and the 4-lanes SHA-256 $(j=4)$ requires $[1024 /(4 \times 64)\rceil+1+\lceil(4 \times 32+9) / 64\rceil=8$ Updates. Of course, it is possible to use the 8-lanes SHA-256 on this architecture, but we can only parallelize 4 Updates using the xmm registers. Therefore, the 8-lanes SHA-256 $(j=8)$ on the AVX architecture (where $S=4)$ requires $[1024 /(4$ $\times 64)\rceil+1+\lceil(8 \times 32+9) / 6\rceil=10$ Updates. 
Figures 2-4 show the number of Update calls (some are parallelized). As seen on Figure 2, when the number of lanes is limited by the SIMD architecture, the total number of Updates for the different choices of $j$, varies only by the number of Updates that are required by the final wrapping stage.

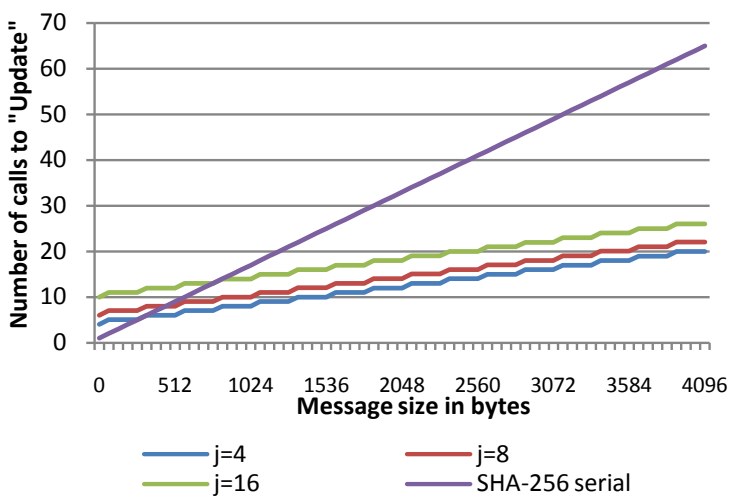

Figure 2. The number of serially computed Updates required on a SIMD architecture supporting 4 lanes (e.g., AVX on a Sandy Bridge architecture), for different message lengths and different choices of $j$.

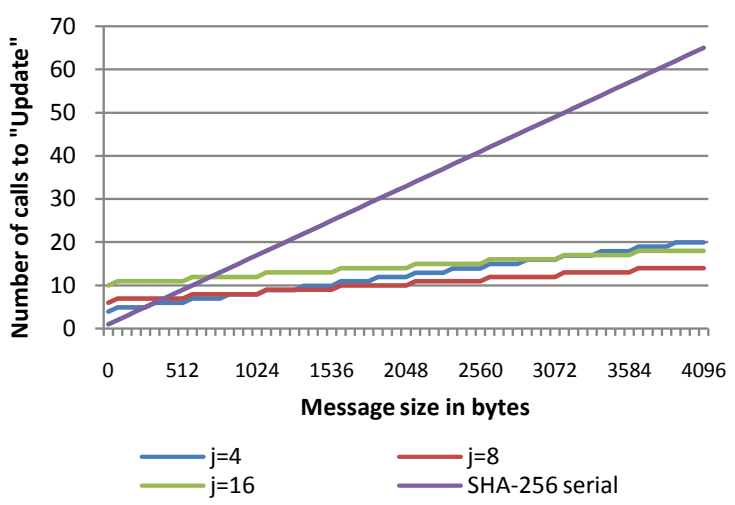

Figure 3. The number of serially computed Updates required on a SIMD architecture supporting 8 lanes (e.g., AVX2 on a Haswell architecture), for different message lengths and different choices of $j$.

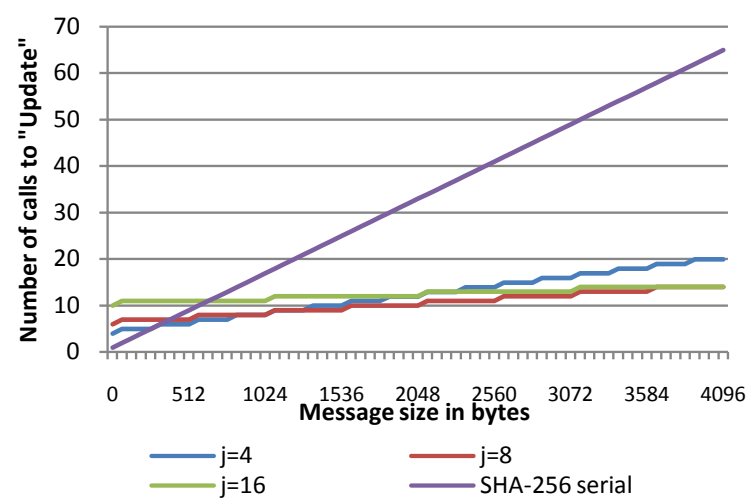

Figure 4. The number of serially computed Updates required on a SIMD architecture supporting 16 lanes (AVX512f - a future architecture), for different message lengths and different choices of $j$. 
However, in Figure 4, we see the differences when the choice of $j=16$ becomes the most efficient for message sizes of $4 \mathrm{~KB}$ and up, requiring the fewest Updates. For $4 \mathrm{~KB}$ messages, both $j=16$ and $j=8$ require 14 Updates, $j=4$ requires 20 updates and the serial SHA-256 requires 65 Updates.

\section{The $j$-Lanes Hash and the $j$-Pointers Hash with Different IVs}

The Merkle-Damgård construction uses one $d$-bit IV to initialize the computations. For $j$-lanes hashing, one might prefer to modify the IVs and this section proposes a method to achieve that.

Define $j+1$ "Prefix" blocks ("Pre") as follows:

$$
\text { Pre }_{i}=j\|i\| \text { type } \| \text { HASH } \| 0^{B-N C H A R-9} i=0,1 \ldots, j
$$

where

- $j$ is encoded as a 32-bit integer in little-endian notation.

- $i$ in the "index" of the lane, and is encoded as a 32-bit integer in little-endian notation. The values $i=0, \ldots, j$ -1 are used for the lanes, and the value $i=j$ is used for the wrapping step.

- type is a single byte with the value $0 \mathrm{x} 0$ for a $j$-lanes hash, and $0 \mathrm{x} 1$ for a $j$-pointers hash.

- HASH is the name of the underlying hash function, encoded as a string of ASCII characters. For SHA-256 we write HASH = "SHA256" or, as ASCII, 0x53, 0x48, 0x41, 0x32, 0x35, 0x36 (encoding "S" = 0x53, "H" $=0 \times 48$, "A" $=0 \times 41$ etc.).

- The number of characters (NCHAR) in the string that indicates HASH should be such that $N C H A R+9 \leq B$.

The Prefix blocks are prepended to the $j+1$ hashed messages, and modify the "effective" IV that is being used. In other words, the $j$-lanes algorithm executes the following computations:

$$
\begin{aligned}
& \mathrm{H}_{0}=\text { HASH }\left(\text { Pre }_{0} \| \text { Buff }_{0}, \text { length }\left(\text { Buff }_{0}\right)+\mathrm{B}\right) \\
& \mathrm{H}_{1}=\text { HASH }\left(\text { Pre }_{1} \| \text { Buff }_{1} \text {, length }\left(\text { Buff }_{1}\right)+\mathrm{B}\right) \\
& \mathrm{H}_{2}=\text { HASH }\left(\text { Pre }_{2} \| \text { Buff }_{2} \text {, length }\left(\text { Buff }_{2}\right)+\mathrm{B}\right) \\
& \ldots \\
& \mathrm{H}_{j-1}=\text { HASH }\left(\text { Pre }_{j-1} \| \text { Buff }_{j-1}, \text { length }\left(\text { Buff }_{j-1}\right)+\mathrm{B}\right) \\
& \mathrm{H}=\mathrm{HASH}\left(\text { Pre }_{j}\left\|\mathrm{H}_{0}\right\| \ldots \| \mathrm{H}_{j-1}, j \times \mathrm{D}+\mathrm{B}\right)
\end{aligned}
$$

Remark 5: SHA-256 allows hashing a message of any length less than $2^{64}$ bits. In the $j$-lanes $j$-pointers modes, the length of the message should be less than $2^{64}-512$ bits.

\section{Pre-Computing the IVs}

The Prefix blocks do not need to be re-computed for each message. Instead, the $j+1$ IV values can be precomputed by:

$$
I V_{i}=\text { compress }\left(\operatorname{Hash} I V, \text { Pre }_{i}\right) ; i=0,1 \ldots, j
$$

Note that the Prefix blocks can also be viewed as a modification of HASH, to use the new IVs instead of a fixed IV. For convenience, denote the hash function that uses $\mathrm{IV}_{i}$ by HASH'${ }_{i}$. In that case the SHA-256 padding shall still accommodate the length of the prefix block.

With this notation, the $j$-lanes hashing can be expressed in terms of HASH' by:

$\mathrm{H}_{0}=\mathrm{HASH}_{0}{ }_{0}\left(\right.$ Buff $_{0}$, length $\left(\right.$ Buff $\left.\left._{0}\right)\right)$

$\mathrm{H}_{1}=\mathrm{HASH}_{1}{ }_{1}\left(\right.$ Buff $_{1}$, length $\left(\right.$ Buff $\left.\left._{1}\right)\right)$

$\mathrm{H}_{2}=\mathrm{HASH}_{2}\left(\right.$ Buff $_{2}$, length $\left(\right.$ Buff $\left.\left._{2}\right)\right)$

...

$\mathrm{H}_{j-1}=\mathrm{HASH}_{j-1}{ }_{j}\left(\right.$ Buff $_{j-1}$, length $\left(\right.$ Buff $\left.\left._{j-1}\right)\right)$

$\mathrm{H}=\mathrm{HASH}_{j}{ }_{j}\left(\mathrm{H}_{0}\left\|\mathrm{H}_{1}\right\| \mathrm{H}_{2}\|\ldots\| \mathrm{H}_{j-1}, j \times \mathrm{D}\right)$

Figure 5 shows the values of the prefix blocks and the new IVs (for HASH = SHA-256).

Remark 5: the following alternative can be considered, for saving the space of storing $j+1$ IV values. Instead, use a single (new) IV value for all the $j+1$ hash computations. We fixed one value of $i d x$, namely $i d x=j$ +1 , and define the $j$-lanes hash by:

$$
\begin{aligned}
& \mathrm{H}_{0}=\mathrm{HASH}^{\prime}{ }_{\mathrm{j}+1}\left(\text { Buff }_{0} \text {, length }\left(\text { Buff }_{0}\right)\right) \\
& \mathrm{H}_{1}=\mathrm{HASH}^{\prime}{ }_{\mathrm{j}+1}\left(\text { Buff }_{1} \text {, length }\left(\text { Buff }_{1}\right)\right) \\
& \ldots
\end{aligned}
$$




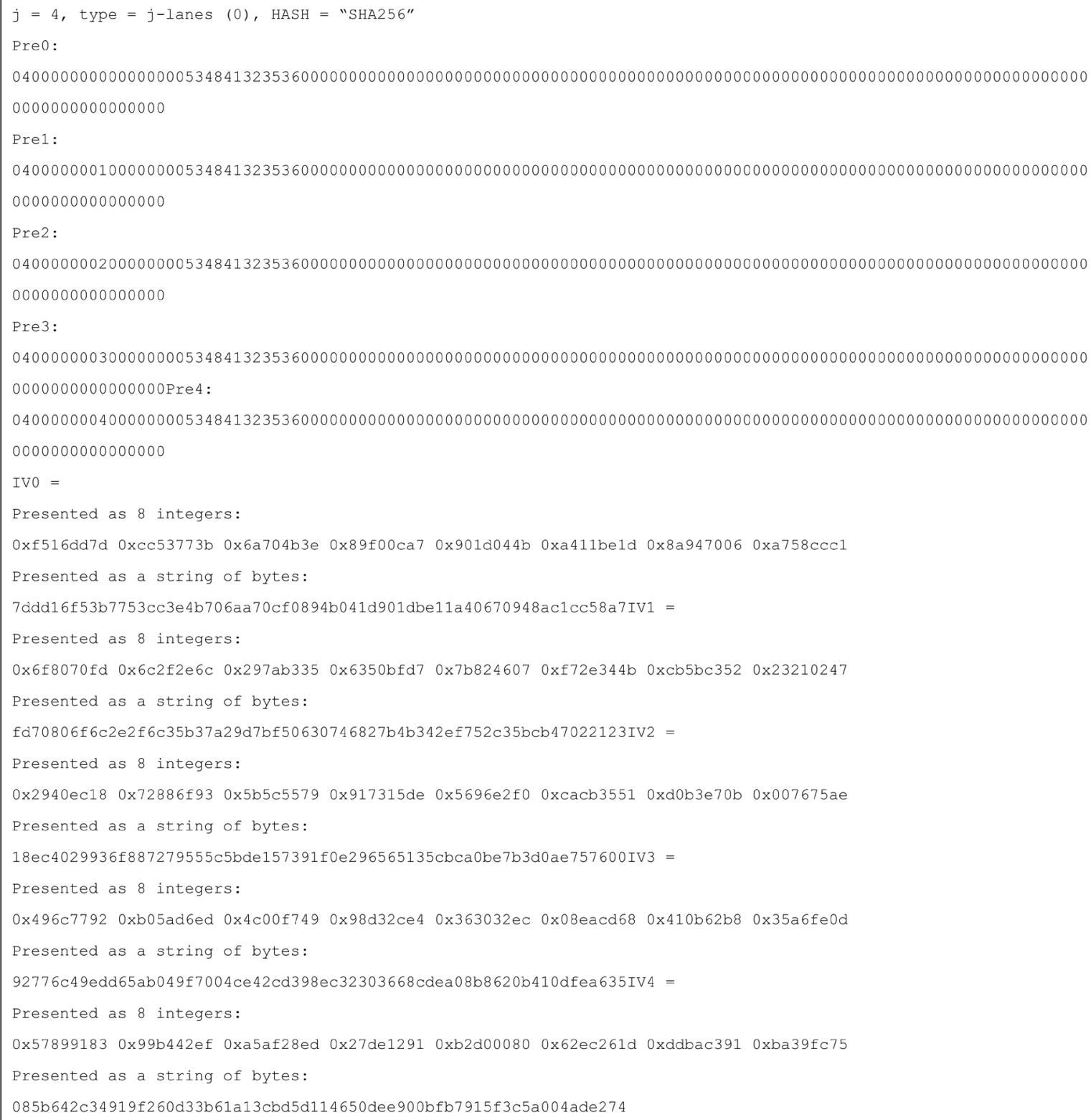

Figure 5. An example for the Prefix blocks and the IVs generation for the 4-lanes SHA-256 hash function.

$\mathrm{H}_{j-1}=\mathrm{HASH}^{\prime}{ }_{j+1}\left(\right.$ Buff $_{j-1}$, length $\left(\right.$ Buff $\left.\left._{j-1}\right)\right)$

$\mathrm{H}=\mathrm{HASH}^{\prime}{ }_{j+1}\left(\mathrm{H}_{0}\left\|\mathrm{H}_{1}\right\| \mathrm{H}_{2}\|\ldots\| \mathrm{H}_{j-1}, j \times \mathrm{D}\right)$

Figure 6 shows the values of the prefix block and the new IV (for HASH = SHA-256) for the alternative.

Test vectors for $j$-lanes SHA-256 with $j=4,8,16$ are provided in the Appendix.

\section{Performance}

This section shows the measured performance of $j$-lanes SHA-256, for $j=4,8,16$, and compares it to the performance of the serial implementation of SHA-256. The results are shown in Figure 7 and Figure 8.

Clearly, the $j$-lanes SHA-256 has a significant performance advantage over the serial SHA-256, for messages that are at least a few kilobytes long. The choice of $j$ affects the hashing efficiency: for a given architecture, $j$-lanes SHA-256 with $j>S$ is slower than $j$-lanes SHA-256 with the optimal choice of $j=S$, due to the longer wrapping step. However, the differences become almost negligible for long messages. 


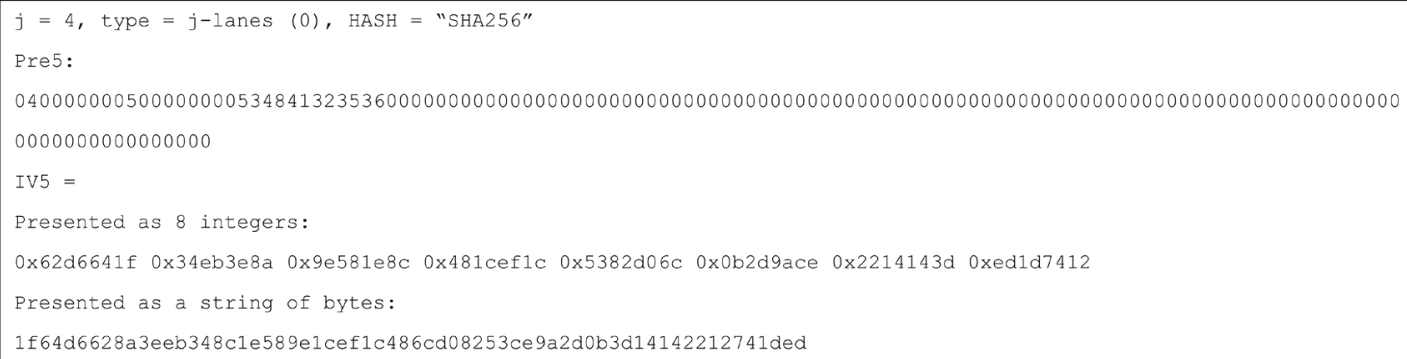

Figure 6. An example of the Prefix block and the (single) IV generation, for the 4-lanes, SHA-256 hash function, for the variant that uses only one modified IV.

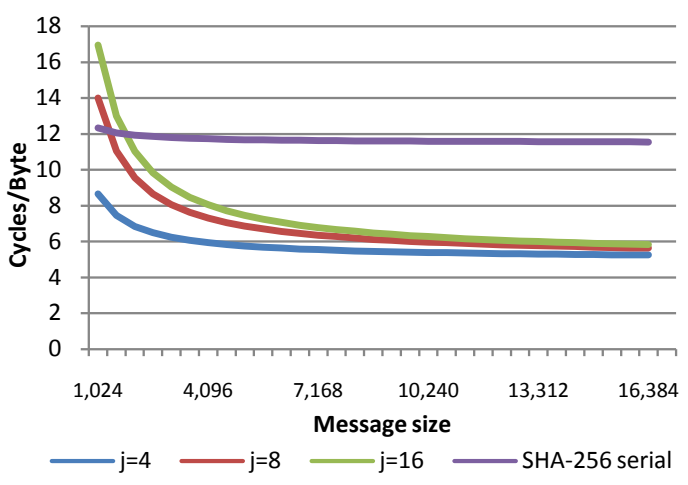

Figure 7. Performance of SHA-256 $j$-lanes compared to the serial SHA-256 implementation, Intel Architecture Codename Sandy Bridge $(S=4)$.

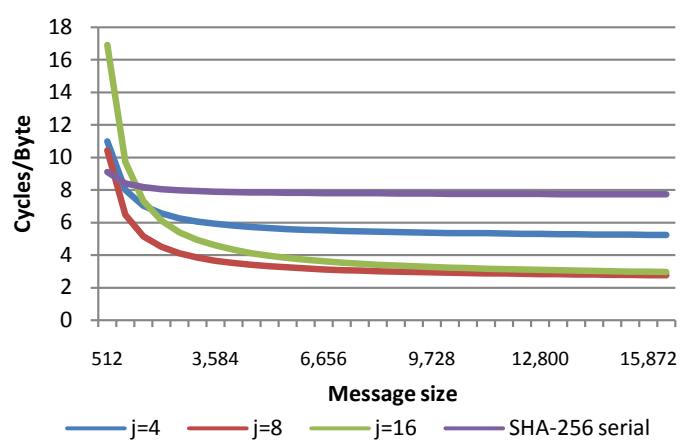

Figure 8. Performance of SHA-256 $j$-lanes compared to the serial SHA-256 implementation, Intel Architecture Codename Haswell $(S=8)$.

\section{Conclusions}

This paper showed the advantages of a $j$-lanes hashing method on modern processors, and provided information on how it can be easily defined and standardized.

The choice of $j$ is a point that needs discussion. If a standard supports different $j$ values, then the optimal choice can be selected per platform. This, however, could add an interoperability burden, and we can imagine that a single value of $j$ would be preferable. In this context, we point out that Figure 2 and Figure 3 (theoretical approximations) are consistent with Figure 7 and Figure 8 for $j=4$ and $j=8$ (actual measurements). Therefore, Figure 4 can be viewed as a good indication for what can be expected when using $j=16$ on the future architectures that would introduce the AVX512f architecture (supporting $S=16$ ). Furthermore, $j=16$ allows better parallelization on multicore platforms. Consequently, our conclusion is that if only one value of $j$ is to be specified by a standard, then the choice of $j=16$ would be the most advantageous. 


\section{References}

[1] Gueron, S. (2013) A j-Lanes Tree Hashing Mode and j-Lanes SHA-256. Journal of Information Security, 4, 7-11.

[2] FIPS (2012) Secure Hash Standard (SHS), Federal Information Processing Standards Publication 180-4. http://csrc.nist.gov/publications/fips/fips180-4/fips-180-4.pdf

[3] Intel (2013) Intel ${ }^{\circledR}$ Architecture Instruction Set Extensions Programming Reference. http://software.intel.com/en-us/file/319433-017pdf

[4] ARM (2013) Neon, ARM. http://www.arm.com/products/processors/technologies/neon.php

[5] Reinders, J. (2013) AVX-512 Instructions, Intel Developer Zone. http://software.intel.com/en-us/blogs/2013/avx-512-instructions 


\section{Appendix: Test Vectors}

The test vectors provided below use the same 1024 bytes message $(M)$ that is defined by (Figures 9 -12). uint8_t M[1024];

for (int $\mathrm{i}=0 ; \mathrm{i}<512 ; \mathrm{i}++)\{\mathrm{M}[\mathrm{i} * 2]=\mathrm{i}>>8$; $\mathrm{M}[\mathrm{i} * 2+1]=\mathrm{i} \& 0 \times \mathrm{ff} ;\}$

The message M (1024 bytes):

$0000000100020003000400050006000700080009000 a 000 b 000 c 000 d 000$ e000f0010001100120013001400 $150016001700180019001 \mathrm{a} 001 \mathrm{~b} 001 \mathrm{c} 001 \mathrm{~d} 001 \mathrm{e} 001 \mathrm{f} 0020002100220023002400250026002700280029002 \mathrm{a}$ $002 b 002 \mathrm{c} 002 \mathrm{~d} 002 \mathrm{e} 002 f 0030003100320033003400350036003700380039003 \mathrm{a} 003 \mathrm{~b} 003 \mathrm{c} 003 \mathrm{~d} 003 e 003 f 00$ $40004100420043004400450046004700480049004 \mathrm{a} 004 \mathrm{~b} 004 \mathrm{c} 004 \mathrm{~d} 004 \mathrm{e} 004 \mathrm{f} 005000510052005300540055$ $0056005700580059005 a 005 b 005 c 005 d 005 e 005 f 0060006100620063006400650066006700680069006 a 00$ 6b006c006d006e006f0070007100720073007400750076007700780079007a007b007c007d007e007f0080 $008100820083008400850086008700880089008 a 008 b 008 c 008 d 008 e 008 f 00900091009200930094009500$ 96009700980099009a009b009c009d009e009f00a000a100a200a300a400a500a600a700a800a900aa00ab 00ac00ad00ae00af00b000b100b200b300b400b500b600b700b800b900ba00bb00bc00bd00be00bf00c000 $c 100 c 200 c 300 c 400 c 500 c 600 c 700 c 800 c 900 c a 00 c b 00 c c 00 c d 00 c e 00 c f 00 d 000 d 100 d 200 d 300 d 400 d 500 d 6$ 00d700d800d900da00db00dc00dd00de00df00e000e100e200e300e400e500e600e700e800e900ea00eb00 ec00ed00ee00ef00f000f100f200f300f400f500f600f700f800f $900 f a 00 f b 00 f c 00 f d 00 f e 00 f f 01000101$ 01020103010401050106010701080109010 a010b010c010d010e010f011001110112011301140115011601 $1701180119011 \mathrm{a} 011 \mathrm{~b} 011 \mathrm{c} 011 \mathrm{~d} 011 \mathrm{e} 011 \mathrm{f} 0120012101220123012401250126012701280129012 \mathrm{a} 012 \mathrm{~b} 012 \mathrm{c}$ 012d012e012f0130013101320133013401350136013701380139013a013b013c013d013e013f0140014101 420143014401450146014701480149014 a 014b014c014d014e014f01500151015201530154015501560157 $01580159015 a 015 b 015 c 015 d 015 e 015 f 0160016101620163016401650166016701680169016 a 016 b 016 c 01$ 6d016e016f0170017101720173017401750176017701780179017a017b017c017d017e017f018001810182 $0183018401850186018701880189018 \mathrm{a} 018 \mathrm{~b} 018 \mathrm{c} 018 \mathrm{~d} 018 \mathrm{e} 018$ f0190019101920193019401950196019701 980199019a019b019c019d019e019f01a001a101a201a301a401a501a601a701a801a901aa01ab01ac01ad 01ae01af01b001b101b201b301b401b501b601b701b801b901ba01bb01bc01bd01be01bf01c001c101c201 c301c401c501c601c701c801c901ca01cb01cc01cd01ce01cf01d001d101d201d301d401d501d601d701d8 01d901da01db01dc01dd01de01df01e001e101e201e301e401e501e601e701e801e901ea01eb01ec01ed01 ee01ef01f001f101f201f301f401f501f601f701f801f901fa01fb01fc01fd01fe01ff

Figure 9. The message M used for the test vectors. 


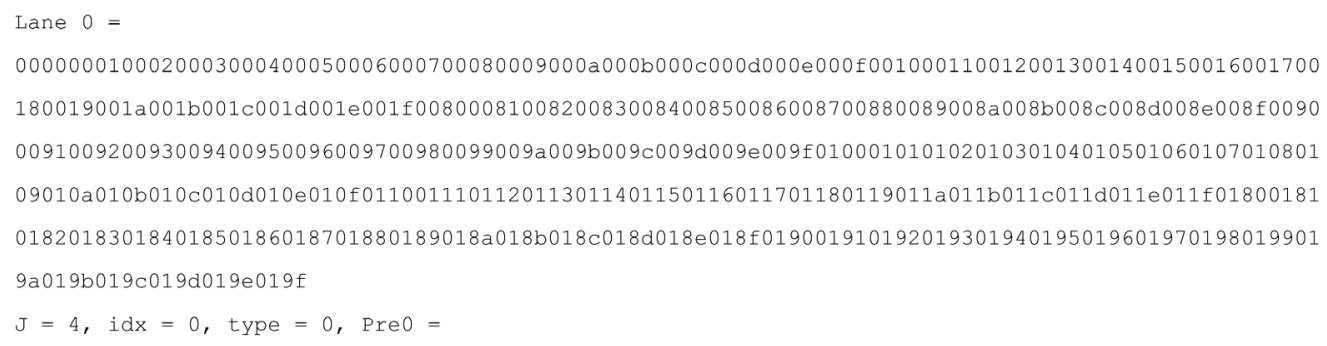


000000000000000000000000000000

IV2 =

Presented as 8 integers:

0x2940ec18 0x72886f93 0x5b5c5579 0x917315de 0x5696e2fo 0xcacb3551 0xd0b3e70b 0x007675ae

Presented as a string of bytes:

$18 e c 4029936$ f887279555c5bde157391f0e296565135cbca0be7b3d0ae757600

$\mathrm{H} 2=$

Presented as 8 integers:

0xa5bff793 0x54e0b9c7 0x38a4abf5 0xf51d6858 0xd4786561 0x51b0b779 0xf92c6680 0x62962ae6

Presented as a string of bytes:

93f7bfa5c7b9e054f5aba43858681df5616578d479b7b05180662cf9e62a9662

Lane $3=$

$0060006100620063006400650066006700680069006 \mathrm{a} 006 \mathrm{~b} 006 \mathrm{c} 006 \mathrm{~d} 006 \mathrm{e} 006 \mathrm{f} 0070007100720073007400750076007700$ $780079007 a 007 b 007 c 007 d 007 e 007 f 00 e 000 e 100 e 200 e 300 e 400 e 500 e 600 e 700 e 800 e 900$ ea 00 eb 00 ec 00 ed 00 ee 00 ef 00 f 0 $00 £ 100 £ 200 £ 300 f 400 f 500 £ 600 £ 700 £ 800 f 900 f a 00 f b 00 f c 00 £ d 00 f e 00 £ f 01600161016201630164016501660167016801$ 69016a016b016c016d016e016f0170017101720173017401750176017701780179017a017b017c017d017e017f01e001e1 01e201e301e401e501e601e701e801e901ea01eb01ec01ed01ee01ef01f001f101f201f301f401f501f601f701f801f 901 fa01fb01fc01fd01fe01ff

$\mathrm{J}=4, i d x=3$, type $=0$, Pre3 $=$

04000000030000000053484132353600000000000000000000000000000000000000000000000000000000000000000000 000000000000000000000000000000

IV3 $=$

Presented as 8 integers:

0x496c7792 0xb05ad6ed 0x4c00f749 0x98d32ce4 0x363032ec 0x08eacd68 0x410b62b8 0x35a6fe0d

Presented as a string of bytes:

$92776 \mathrm{c} 4$ 9edd65ab049f7004ce42cd398ec32303668cdea08b8620b410dfea635

$\mathrm{H} 3=$

Presented as 8 integers:

0xda669dfe 0x86fabd5e 0xc9bacdf8 0x1452d42d 0x51daf0a3 0x0e072407 0x4b1e0240 0xc5b4fd16

Presented as a string of bytes:

fe9d66da5ebdfa86f8cdbac92dd45214a3f0da510724070e40021e4b16fdb4c5

The wrapping string (the concatenation of $j$ digests) $=$

a291b60c1c93e74c55901e2ba918a5b6809ae2b58de7f79629a6f9be3166231cfb4c3a01163982a802a6c26dfd24db111a e3b4c2f9f50862ef9809elff2a25c393f7bfa5c7b9e054f5aba43858681df5616578d479b7b05180662cf9e62a9662fe9d $66 \mathrm{da} 5 \mathrm{ebdfa} 86 \mathrm{f} 8 \mathrm{cdbac} 92 \mathrm{dd} 45214 \mathrm{a} 3 \mathrm{f} 0 \mathrm{da} 510724070 \mathrm{e} 40021 \mathrm{e} 4 \mathrm{~b} 16 \mathrm{fdb} 4 \mathrm{c5}$

$\mathrm{J}=4, i d x=4$, type $=0$, Pre4 $=$

04000000040000000053484132353600000000000000000000000000000000000000000000000000000000000000000000 000000000000000000000000000000

IV4 =

Presented as 8 integers:

0x57899183 0x99b442ef 0xa5af28ed 0x27de1291 0xb2d00080 0x62ec261d 0xddbac391 0xba39fc75

Presented as a string of bytes:

83918957 ef42b499ed28afa59112de278000d0b21d26ec6291c3badd75fc39ba

The output digests, $\mathrm{H}=$

Presented as 8 integers:

0x2c645b08 0x269f9134 0x1ab6330d 0xd1d5cb13 0xee0d6514 0x79fb0b90 0xa0c5f315 0x74e2ad04

Presented as a string of bytes:

085b642c34919f260d33b61a13cbd5d114650dee 900 bfb $7915 £ 3$ c5a 004 ade2 74

Figure 10. Test vector for SHA-256 4-lanes. 
Lane $0=$

$0000000100020003000400050006000700080009000 a 000$ b000c000d000e000f0010001100120013001400 $150016001700180019001 \mathrm{a} 001 \mathrm{~b} 001 \mathrm{c} 001 \mathrm{~d} 001 \mathrm{e} 001 \mathrm{f} 0100010101020103010401050106010701080109010 \mathrm{a}$ 010b010c010d010e010f0110011101120113011401150116011701180119011a011b011c011d011e011f $\mathrm{J}=8, i \mathrm{dx}=0$, type $=0, \operatorname{Pre} 0=$

08000000000000000053484132353600000000000000000000000000000000000000000000000000000000 000000000000000000000000000000000000000000

IVO $=$

Presented as 8 integers:

0x787f6051 0x684c02c0 0xde7ccd48 0x2c6382de 0x903f8cc0 0x74c60570 0xd8e5e679 0xfcad483d Presented as a string of bytes:

$51607 f 78 c 0024 c 6848 c d 7 c d e d e 82632 c c 08 c 3 f 907005 c 67479 e 6 e 5 d 83 d 48 a d f c$

$\mathrm{H} 0=$

Presented as 8 integers:

0xd90a9208 0xb1cd8603 0x967e141c 0x9dc938f7 0x28005edc 0x549a7429 0xac6c2d6f 0x576bd8b1 Presented as a string of bytes:

$08920 a d 90386 c d b 11 c 147 e 96 f 738 c 99 d d c 5 e 002829749 a 546 f 2 d 6 c a c b 1 d 86 b 57$

Lane $1=$

$0020002100220023002400250026002700280029002 a 002 b 002 c 002 d 002 e 002 f 0030003100320033003400$ $350036003700380039003 a 003 b 003 c 003 d 003 e 003 f 0120012101220123012401250126012701280129012 a$ 012b012c012d012e012f0130013101320133013401350136013701380139013a013b013c013d013e013f

$\mathrm{J}=8, i d x=1$, type $=0$, Pre $1=$

08000000010000000053484132353600000000000000000000000000000000000000000000000000000000 000000000000000000000000000000000000000000

IV1 $=$

Presented as 8 integers:

0x39fa5544 0x74d24640 0xf0435922 0xcdlf50b4 0xdfd3eaf6 0x4f295f3a 0xcebedb2a 0xe3126408 Presented as a string of bytes:

$4455 f a 394046 \mathrm{~d} 274225943 f 0 b 4501 \mathrm{fcdf} 6 \mathrm{ead} 3 \mathrm{df} 3 \mathrm{a} 5 \mathrm{f} 294 \mathrm{f} 2 \mathrm{adbbece} 086412 \mathrm{e} 3$

$\mathrm{H} 1=$

Presented as 8 integers:

0x86753c04 0xa3825b56 0xd9dcaa47 0xf84d0f91 0x1b412197 0x1135f42b 0x953a6ba1 0x30d5f9b4 Presented as a string of bytes:

043c7586565b82a347aadcd9910f4df89721411b2bf43511a16b3a95b4f9d530

Lane $2=$

$0040004100420043004400450046004700480049004 a 004 b 004 \mathrm{c} 004 \mathrm{~d} 004 \mathrm{e} 004 \mathrm{f} 0050005100520053005400$ $550056005700580059005 a 005 b 005 c 005 d 005 e 005 f 0140014101420143014401450146014701480149014 a$ $014 \mathrm{~b} 014 \mathrm{c} 014 \mathrm{~d} 014 \mathrm{e} 014 f 0150015101520153015401550156015701580159015 \mathrm{a} 015 \mathrm{~b} 015 \mathrm{c} 015 \mathrm{~d} 015 \mathrm{e} 015 \mathrm{f}$

$\mathrm{J}=8, i d x=2$, type $=0, \operatorname{Pre} 2=$ 
08000000020000000053484132353600000000000000000000000000000000000000000000000000000000 000000000000000000000000000000000000000000

IV $2=$

Presented as 8 integers:

0x6662dd71 0x809cbd72 0x3fe09a5f 0xb75372fa 0x87ef7577 0x7e317792 0x010d9ccf 0xb474ba3b

Presented as a string of bytes:

71dd626672bd9c805f9ae03ffa7253b77775ef879277317ecf9c0d013bba74b4

$\mathrm{H} 2=$

Presented as 8 integers:

0x5181afc2 0x8118f2e6 ox054e5ab0 0xcf2d001e 0x15ad7615 0x6e57d085 ox49d20875 0x315f1180

Presented as a string of bytes:

c2af8151e6f21881b05a4e051e002dcf1576ad1585d0576e7508d24980115f31

Lane $3=$

$0060006100620063006400650066006700680069006 a 006 b 006 c 006 d 006 e 006 f 0070007100720073007400$ $750076007700780079007 a 007 b 007 c 007 d 007 e 007 f 0160016101620163016401650166016701680169016 \mathrm{a}$ 016b016c016d016e016f0170017101720173017401750176017701780179017a017b017c017d017e017f

$\mathrm{J}=8, i \mathrm{dx}=3$, type $=0, \operatorname{Pre} 3=$

08000000030000000053484132353600000000000000000000000000000000000000000000000000000000 000000000000000000000000000000000000000000

IV $3=$

Presented as 8 integers:

0x11ae33b4 0x5dc9d2c8 0xa5f61621 0x48adaed6 0x4baf1946 0xf0092642 0x9202b4da 0x023111fe Presented as a string of bytes:

b433ae11c8d2c95d2116f6a5d6aead484619af4b422609f0dab40292fe113102

H3 $=$

Presented as 8 integers:

0xbe9d443c 0x4a550840 0xb28919fd 0x52502fa2 0x226211c8 0x911f847c 0xb97ca0a5 0xa20e2a6a

Presented as a string of bytes:

3c449dbe4008554afd1989b2a22f5052c81162227c841f91a5a07cb96a2a0ea2

Lane $4=$

$0080008100820083008400850086008700880089008 \mathrm{a} 008 \mathrm{~b} 008 \mathrm{c} 008 \mathrm{~d} 008 \mathrm{e} 008 \mathrm{f} 0090009100920093009400$ $950096009700980099009 a 009 b 009 \mathrm{c} 009 d 009 e 009 f 0180018101820183018401850186018701880189018 \mathrm{a}$ 018b018c018d018e018f0190019101920193019401950196019701980199019a019b019c019d019e019f $\mathrm{J}=8, i \mathrm{dx}=4$, type $=0$, Pre $4=$

08000000040000000053484132353600000000000000000000000000000000000000000000000000000000 000000000000000000000000000000000000000000

IV $4=$

Presented as 8 integers:

0x08ed680a 0xc788f0c0 0x497d599e 0x79c12056 0xf9435c3d 0x28bdd5b0 0xd953912f 0xbe006e9f 


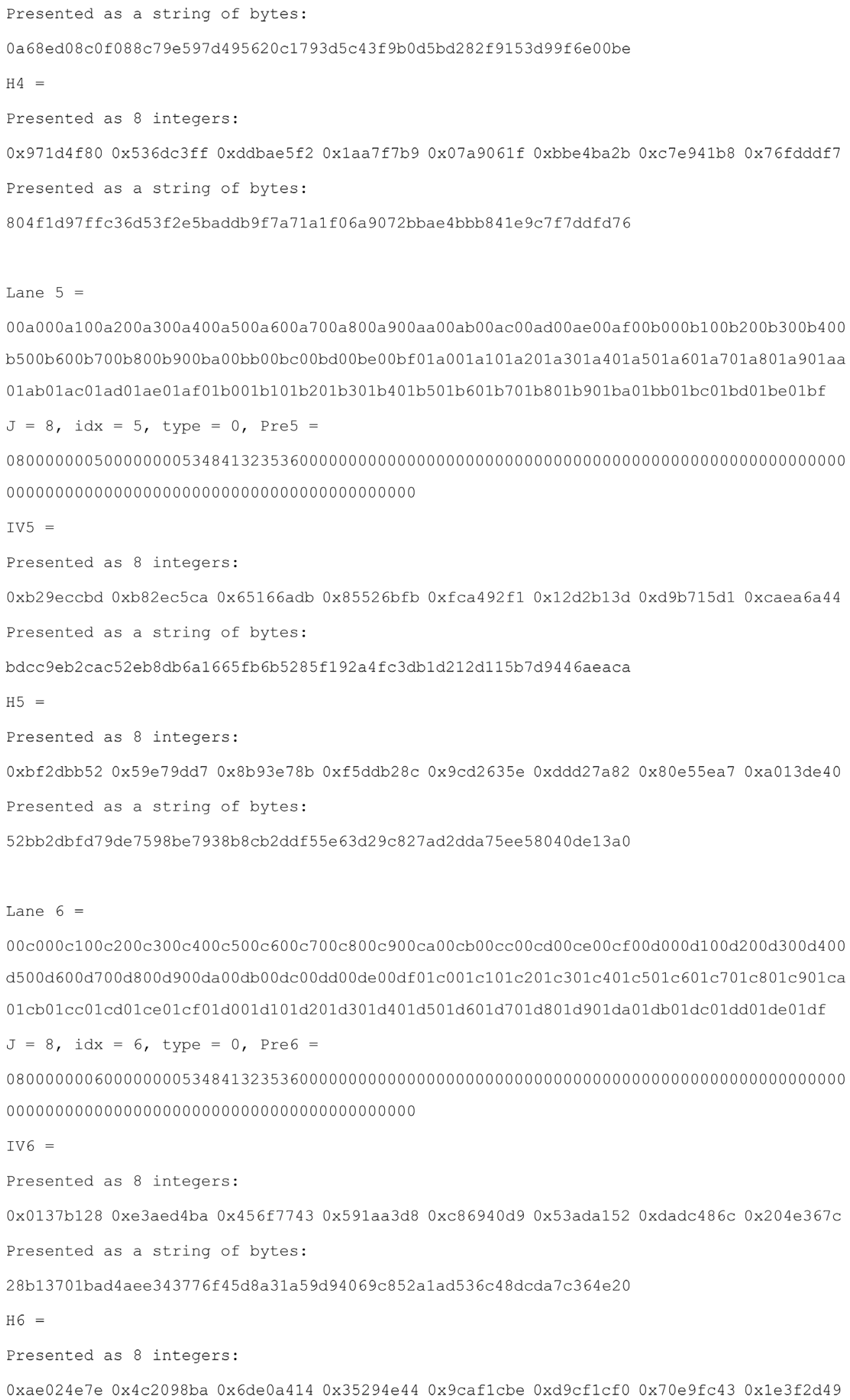




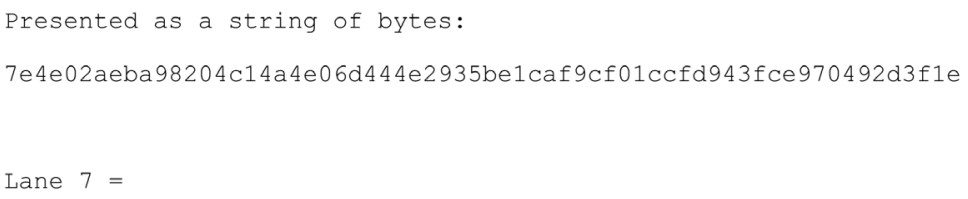

Figure 11. Test vector for SHA-256 8-lanes. 
Lane $0=$

$0000000100020003000400050006000700080009000 a 000 b 000 c 000 d 000 e 000 f 0010001100120013001400$ $150016001700180019001 \mathrm{a} 001 \mathrm{~b} 001 \mathrm{c} 001 \mathrm{~d} 001 \mathrm{e} 001 \mathrm{f}$

$\mathrm{J}=16, i \mathrm{dx}=0$, type $=0$, Pre $0=$

10000000000000000053484132353600000000000000000000000000000000000000000000000000000000 000000000000000000000000000000000000000000

IV0 $=$

Presented as 8 integers:

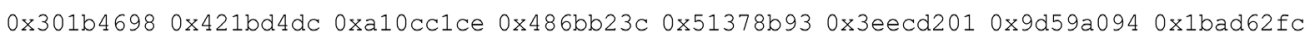
Presented as a string of bytes:

98461b30dcd41b42cec10ca13cb26b48938b375101d2ec3e94a0599dfc62ad1b

H0 =

Presented as 8 integers:

0x472d67f1 0x3ed556ca 0x88516bfb 0x0adaae63 0x43af34ce 0x0353eaab 0xb01635dc 0x3af7d38d Presented as a string of bytes:

f1672d47ca56d53efb6b518863aeda0ace34af43abea5303dc3516b08dd3f73a

Lane $1=$

$0020002100220023002400250026002700280029002 a 002 b 002 c 002 d 002 e 002 f 0030003100320033003400$ $350036003700380039003 a 003 b 003 c 003 d 003 e 003 f$

$\mathrm{J}=16, i \mathrm{dx}=1$, type $=0$, Pre1 $=$

10000000010000000053484132353600000000000000000000000000000000000000000000000000000000 000000000000000000000000000000000000000000

IV1 $=$

Presented as 8 integers:

0xe584be20 0x6be7e96c 0x59d99116 0xcd3cd22e 0xbb9d678c 0x782af33d. 0xacec61ac 0x6eedcea4 Presented as a string of bytes:

20be84e56ce9e76b1691d9592ed23ccd8c679dbb3df32a78ac61ecaca4ceed6e

$\mathrm{H} 1=$

Presented as 8 integers:

0x383545a8 0xebccaf84 0xe25793bf 0xaf8d34d3 0x8f07e023 0xf71a2ab5 0x663d4152 0x8798c2ef Presented as a string of bytes:

a845353884afccebbf9357e2d3348daf23e0078fb52alaf752413d66efc29887

Lane $2=$

$0040004100420043004400450046004700480049004 a 004 b 004 c 004 d 004 e 004 f 0050005100520053005400$ $550056005700580059005 \mathrm{a} 005 \mathrm{~b} 005 \mathrm{c} 005 \mathrm{~d} 005 \mathrm{e} 005 \mathrm{f}$

$\mathrm{J}=16$, $i \mathrm{dx}=2$, type $=0$, Pre2 $=$

10000000020000000053484132353600000000000000000000000000000000000000000000000000000000 000000000000000000000000000000000000000000

IV2 =

Presented as 8 integers:

0x83d278b8 0x5b8e8240 0x2b4df96c 0xafbeb60a 0xf1a519ee 0xab829302 0xe518a3d9 0x55be0fbd Presented as a string of bytes:

b8 $78 d 28340828$ e 5 b 6 cf $94 d 2 b 0 a b 6$ beafee $19 a 5 f 1029382 a b d 9 a 318$ e 5 bdofbe 55

$\mathrm{H} 2=$

Presented as 8 integers:

0x824307a9 0xda8acc6c 0xffeb1ed6 0xf3315f02 0xb4bb635e 0xafee9d3d 0xbcc2b49d 0x42c3daae Presented as a string of bytes:

a $90743826 \mathrm{ccc} 8 \mathrm{adad} 61$ eebff $025 \mathrm{f} 31 \mathrm{f} 35 \mathrm{e} 63 \mathrm{bbb} 43 \mathrm{~d} 9 \mathrm{deeaf} 9 \mathrm{db} 4 \mathrm{c} 2 \mathrm{bcaedac} 342$ 
Lane $3=$

$0060006100620063006400650066006700680069006 \mathrm{a} 006 \mathrm{~b} 006 \mathrm{c} 006 \mathrm{~d} 006 \mathrm{e} 006 \mathrm{f} 0070007100720073007400$ $750076007700780079007 a 007 b 007 c 007 d 007 e 007 f$

$\mathrm{J}=16, i \mathrm{dx}=3$, type $=0$, Pre3 $=$

10000000030000000053484132353600000000000000000000000000000000000000000000000000000000 000000000000000000000000000000000000000000

IV3 $=$

Presented as 8 integers:

0x1b5600c7 0x3c12c7a0 0x31df144c 0xd105f19d 0xc5106b02 0xeeb323de 0xb808d185 0xfb6f6550 Presented as a string of bytes:

c700561ba0c7123c4c14df319df105d1026b10c5de23b3ee85d108b850656ffb

H3 =

Presented as 8 integers:

0x419b79f5 0x7e42bc10 0xaf93b5a6 0xa07fc24f 0x2441a0c1 0xe8427787 0xaa3a4d22 0x590e2dbb Presented as a string of bytes:

f5799b4110bc427ea6b593af4fc27fa0c1a04124877742e8224d3aaabb2d0e59

Lane $4=$

$0080008100820083008400850086008700880089008 \mathrm{a} 008 \mathrm{~b} 008 \mathrm{c} 008 \mathrm{~d} 008 \mathrm{e} 008 f 0090009100920093009400$ $950096009700980099009 a 009 b 009 c 009 d 009 e 009 f$

$\mathrm{J}=16, i \mathrm{dx}=4$, type $=0$, Pre $4=$

10000000040000000053484132353600000000000000000000000000000000000000000000000000000000 000000000000000000000000000000000000000000

IV4 =

Presented as 8 integers:

0x270865f5 0xda90a5e7 0x004ed5ac 0xb399cf28 0x598b21a3 0x4a1b7bd4 0xb298a277 0x0c9d36d9

Presented as a string of bytes:

f5650827e7a590daacd54e0028cf99b3a3218b59d47b1b4a77a298b2d9369d0c

$\mathrm{H} 4=$

Presented as 8 integers:

0x0bdea332 0x489e23c0 0x489f243d 0x08b4404b 0xfdbda480 0x9f019c35 0x0e98ec17 0x1788130e

Presented as a string of bytes:

$32 \mathrm{a} 3 \mathrm{de} 0 \mathrm{bc02} 39 \mathrm{e} 483 \mathrm{~d} 249 \mathrm{f} 484 \mathrm{~b} 40 \mathrm{~b} 40880 \mathrm{a} 4 \mathrm{bdfd} 359 \mathrm{c} 019$ f17ec980e0e138817

Lane $5=$

00a000a100a200a300a400a500a600a700a800a900aa00ab00ac00ad00ae00af00b000b100b200b300b400 b500b600b700b800b900ba00bb00bc00bd00be00bf

$\mathrm{J}=16$, $i \mathrm{dx}=5$, type $=0$, Pre $5=$

10000000050000000053484132353600000000000000000000000000000000000000000000000000000000 000000000000000000000000000000000000000000

IV5 $=$

Presented as 8 integers:

0x33aeaf06 0x9d5efd54 0xa98aa21e 0x8df52647 0x730bafe4 0x4e3076af 0xe16e9154 0xbd1d7f07 Presented as a string of bytes:

06 afae3354fd5e9d1ea28aa94726f58de4af0b73af76304e54916ee1077f1dbd

H5 =

Presented as 8 integers:

0xb02d93fc 0xe29f0086 0x84fc3565 0x1f300e86 0x1bf43a85 0x71f91ac8 0xd9742ec0 0x179312e5

Presented as a string of bytes: 
fc932db086009fe26535fc84860e301f853af41bc81af971c02e74d9e5129317

Lane $6=$

$00 c 000 c 100 c 200 c 300 c 400 c 500 c 600 c 700 c 800 c 900 c a 00 c b 00 c c 00 c d 00 c e 00 c f 00 d 000 d 100 d 200 d 300 d 400$ d500d600d700d800d900da00db00dc00dd00de00df

$\mathrm{J}=16, \mathrm{idx}=6$, type $=0$, Pre6 $=$

10000000060000000053484132353600000000000000000000000000000000000000000000000000000000 000000000000000000000000000000000000000000

IV6 $=$

Presented as 8 integers:

0x03205e22 0x345b8bdb 0xdf24elff 0x249e2c65 0xa8c30e39 0x77f91a58 0xe1cb85a4 0x3b6c7448 Presented as a string of bytes:

$225 e 2003 \mathrm{db} 8 \mathrm{~b} 5 \mathrm{~b} 34 \mathrm{ffe} 124 \mathrm{df} 652 \mathrm{c} 9 \mathrm{e} 24390 \mathrm{ec} 3 \mathrm{a} 8581 \mathrm{af} 977 \mathrm{a} 485 \mathrm{cbe} 148746 \mathrm{c} 3 \mathrm{~b}$

H6 =

Presented as 8 integers:

0x83af3937 0x7f30aaf8 0x45fb16ed 0x49ef08ca 0x61a19f7a 0x21b2ecc4 0x2676295c 0x22b1cde4 Presented as a string of bytes:

3739af83f8aa307fed16fb45ca08ef497a9fa161c4ecb2215c297626e4cdb122

Lane $7=$

$00 e 000 e 100 e 200$ e300e400e500e600e700e800e900ea 00 eb00ec00ed00ee00efoof $000 f 100 f 200 f 300 f 400$ f500f600f700f800f900fa00fb00fco0fdoofe00ff

$\mathrm{J}=16$, $i \mathrm{dx}=7$, type $=0, \operatorname{Pre} 7=$

10000000070000000053484132353600000000000000000000000000000000000000000000000000000000 000000000000000000000000000000000000000000

IV7 $=$

Presented as 8 integers:

0x4744b3b3 0xdb42b4ed 0xabe499fb 0xacf298a4 0x929e92ae 0x71c071dc 0xc091cbf5 0xaf33d91f Presented as a string of bytes:

b3b34447edb442dbfb99e4aba498f2acae929e92dc71c071f5cb91c01fd933af

$\mathrm{H} 7=$

Presented as 8 integers:

0x17fc7d87 0x91385485 0x5892e618 0x2fe0f492 0x4914a63d 0x8e3b87f1 0x24f2a715 0x648e0065

Presented as a string of bytes:

$877 \mathrm{dfc} 178554389118 \mathrm{e} 6925892 \mathrm{f} 4 \mathrm{e} 02 \mathrm{f} 3 \mathrm{da} 61449$ f1873b8e15a7f22465008e 64

Lane $8=$

$0100010101020103010401050106010701080109010 a 010$ b010c010d010e010f0110011101120113011401 $150116011701180119011 \mathrm{a} 011 \mathrm{~b} 011 \mathrm{c011d011e011f}$

$\mathrm{J}=16$, idx $=8$, type $=0$, Pre $8=$

10000000080000000053484132353600000000000000000000000000000000000000000000000000000000 000000000000000000000000000000000000000000

IV8 $=$

Presented as 8 integers:

0x88d9773e 0x227b996c 0xb045c986 0x0c6568ca 0x5a31e35f 0xd68a998b 0xa8125b79 0x5e2c81e6

Presented as a string of bytes:

$3 e 77 d 9886 c 997 b 2286 c 945 b 0 c a 68650 c 5 f e 3315 a 8 b 998 a d 6795 b 12 a 8 e 6812 c 5 e$

$\mathrm{H} 8=$

Presented as 8 integers:

0xd1b89671 0x8ce702c4 0xaf5a504c 0xa8c425fa 0x8d44bc0b 0x2407529d 0xb06eeb14 0x4494bc66 
Presented as a string of bytes:

7196b8dlc402e78c4c505aaffa25c4a80bbc448d9d52072414eb6eb066bc9444

Lane $9=$

$0120012101220123012401250126012701280129012 \mathrm{a} 012 \mathrm{~b} 012 \mathrm{c} 012 \mathrm{~d} 012 \mathrm{e} 012 \mathrm{f} 0130013101320133013401$ $350136013701380139013 a 013 b 013 c 013 d 013 e 013 f$

$\mathrm{J}=16, i \mathrm{dx}=9$, type $=0$, Pre9 $=$

10000000090000000053484132353600000000000000000000000000000000000000000000000000000000 000000000000000000000000000000000000000000

IV9 $=$

Presented as 8 integers:

0x6dcb53a7 0x91aa3069 0xf594281f 0x5e3c2a06 0xd9d6474b 0x7b5ff762 0x4a96c1ec 0x1cfdbc57 Presented as a string of bytes:

a753cb6d6930aa911f2894f5062a3c5e4b47d6d962f75f7becc1964a57bcfd1c

H9 $=$

Presented as 8 integers:

0x7aed3aae 0xcc9df426 0x49f39ec1 0xebad8048 0x931b5909 0xd0c15bd9 0x46.bb8d14 0x38daf8f2

Presented as a string of bytes:

ae3aed7a26f4 9dccc19ef3494880adeb09591b93d95bc1d0148dbb46f2f8da38

Lane $10=$

$0140014101420143014401450146014701480149014 a 014 b 014 \mathrm{c} 014 \mathrm{~d} 014 \mathrm{e} 014 \mathrm{f} 0150015101520153015401$ $550156015701580159015 a 015 b 015 c 015 d 015 e 015 f$

$\mathrm{J}=16, i \mathrm{dx}=10$, type $=0$, Pre10 $=$

$100000000 a 0000000053484132353600000000000000000000000000000000000000000000000000000000$ 000000000000000000000000000000000000000000

IV10 =

Presented as 8 integers:

0x6e7be156 0x9a9e3d68 0x835a8de7 0xbc56536d 0x010759e0 0xedale726 0x60cf113a 0xd3b9d737 Presented as a string of bytes:

56e17b6e683d9e9ae78d5a836d5356bce059070126e7aled3a11cf6037d7b9d3

H10 $=$

Presented as 8 integers:

0x66755c29 0xa3a52b52 0xe9d80979 0x1482e24d 0x2c11e40f 0x9302cbc4 0x09b76d4a 0x3816530d Presented as a string of bytes:

$295 \mathrm{c} 7566522 \mathrm{ba} 5 \mathrm{a} 37909 \mathrm{~d} 8 \mathrm{e} 94 \mathrm{de} 282140$ fe4112cc4cb02934a6db7090d531638

Lane $11=$

$0160016101620163016401650166016701680169016 a 016 b 016 c 016 d 016 e 016 f 0170017101720173017401$ 750176017701780179017a017b017c017d017e017f

$\mathrm{J}=16, i \mathrm{dx}=11$, type $=0$, Pre11 $=$

10000000060000000053484132353600000000000000000000000000000000000000000000000000000000 000000000000000000000000000000000000000000

$\operatorname{IV} 11=$

Presented as 8 integers:

0x8cbccae1 0x616bdba3 0xf21abde3 0x551fe2d4 0x0a846720 0x318a5e23 0xcf10df64 0xd3830b54 Presented as a string of bytes:

elcabc8ca3db6b61e3bdlaf2d4e21f552067840a235e8a3164df10cf540b83d3

H11 =

Presented as 8 integers: 
0xf5a975a1 0xc0f9e9e0 0x1a33230f 0x283d99b6 0xdfdf95fb 0x2563b811 0x47e85a61 0x777a92bd

Presented as a string of bytes:

a175a9f5e0e9f9c00£23331ab6993d28fb95dfdf11b86325615ae847bd927a77

Lane $12=$

$0180018101820183018401850186018701880189018 \mathrm{a} 018 \mathrm{~b} 018 \mathrm{c} 018 \mathrm{~d} 018 \mathrm{e} 018 \mathrm{f} 0190019101920193019401$ 950196019701980199019a019b019c019d019e019f

$\mathrm{J}=16, i \mathrm{dx}=12$, type $=0$, Pre12 $=$

10000000000000000053484132353600000000000000000000000000000000000000000000000000000000 000000000000000000000000000000000000000000

IV12 =

Presented as 8 integers:

0xf30aea08 0x6327cb9c 0xe090d724 0xf55belbl 0x356bac3a 0x259e2a90 0x1474593e 0x317eb9f7 Presented as a string of bytes:

08ea0af39ccb276324d790e0b1e15bf53aac6b35902a9e253e597414f7b97e31

H12 =

Presented as 8 integers:

0x60aa4972 0xb64cf665 0x01e11d8d 0x17a2408b 0xd43ad3b3 0x43c75ac6 0xfcb5a860 0x32fa397d Presented as a string of bytes:

$7249 a a 6065 f 64 c b 68 d 1 d e 1018 b 40 a 217 b 3 d 33 a d 4 c 65 a c 74360 a 8 b 5 f c 7 d 39 f a 32$

Lane $13=$

01a001a101a201a301a401a501a601a701a801a901aa01ab01ac01ad01ae01af01b001b101b201b301b401 b501b601b701b801b901ba01bb01bc01bd01be01bf

$\mathrm{J}=16, \mathrm{idx}=13$, type $=0, \operatorname{Pre} 13=$

10000000010000000053484132353600000000000000000000000000000000000000000000000000000000 000000000000000000000000000000000000000000

$\operatorname{IV} 13=$

Presented as 8 integers:

0xeea5ddcb 0xecdc55c4 0xd8554d0a 0xa11207d1 0xe654142b 0xa29989f6 0xdab88e31 0xc66e5305 Presented as a string of bytes:

cbdda 5eec455dcec0a4d55d8d10712a12b1454e6f68999a2318eb8da05536ec6

$\mathrm{H} 13=$

Presented as 8 integers:

0xcc4ea4d6 0xe06b3d10 0x9c7cb023 0x36856036 0x6fb62968 0xd7d29cal 0x62f748f4 0x54b4753f Presented as a string of bytes:

d6a 44 ecc103d6be023b07c9c366085366829b66fa19cd2d7f448f7623f75b454

Lane $14=$

01c001c101c201c301c401c501c601c701c801c901ca01cb01cc01cd01ce01cf01d001d101d201d301d401 d501d601d701d801d901da01db01dc01dd01de01df

$\mathrm{J}=16, i \mathrm{dx}=14$, type $=0, \operatorname{Pre} 14=$

$100000000 e 0000000053484132353600000000000000000000000000000000000000000000000000000000$ 000000000000000000000000000000000000000000

IV14 =

Presented as 8 integers:

0x36c346a5 0x715bbcbc 0xfd5cf994 0x6c4d16b7 0x1a19c99f 0x4c777619 0x412a6300 0x89b25e19 Presented as a string of bytes:

a546c336bcbc5b7194f95cfdb7164d6c9fc9191a1976774c00632a41195eb289

$\mathrm{H} 14=$ 
Presented as 8 integers:

0x1d3bf0a0 0x7d399bf7 0xe64b64c0 0xf7ba2434 0xa409ab5d 0x7870f632 0x82e76833 0xc64bcca1 Presented as a string of bytes:

a0f03b1df79b397dc0644be63424baf75dab09a432f670783368e782a1cc4bc6

Lane $15=$

0le00lelole20le30le40le50le60le70le80le90lea0leb0lec0led0lee0lefolfoolf101f201f301f401 f501f601f701f801f901fa01fb01fC01fd01fe01ff

$\mathrm{J}=16, i \mathrm{dx}=15$, type $=0$, Pre15 $=$

$100000000 f 0000000053484132353600000000000000000000000000000000000000000000000000000000$ 000000000000000000000000000000000000000000

IV15 =

Presented as 8 integers:

Oxe9f3a324 0x739f1383 0x06e52eaf 0x6608f137 0x61e1a290 0x688ae5a8 0xca111224 0x66b3d938 Presented as a string of bytes:

24a3f3e983139f73af2ee50637f1086690a2e161a8e58a68241211ca38d9b366

$\mathrm{H} 15=$

Presented as 8 integers:

0x8d0f2b54 0x743ac7f1 0x4fd432ef 0x56074b6b 0xa9a16d82 0xb61f363a 0x81518dee 0xd7f215cb Presented as a string of bytes:

$542 \mathrm{~b} 0 \mathrm{f} 8 \mathrm{df1c73a} 74 \mathrm{ef} 32 \mathrm{~d} 44 \mathrm{f} 6 \mathrm{~b} 4 \mathrm{~b} 0756826 \mathrm{dala} 93 \mathrm{a} 361 \mathrm{fb} 6 \mathrm{ee} 8 \mathrm{~d} 5181 \mathrm{cb} 15 \mathrm{f} 2 \mathrm{~d} 7$

The wrapping string (the concatenation of $j$ digests) $=$

f1672d47ca56d53efb6b518863aeda 0ace34af43abea5303dc3516b08dd3f73aa845353884afccebbf 9357 e2d3348daf23e0078fb52alaf752413d66efc29887a 90743826 ccc8adad61eebffo 25 f31f35e63bbb43d9d eeaf $9 \mathrm{db} 4 \mathrm{c} 2 \mathrm{bcaedac} 342 \mathrm{f} 5799 \mathrm{~b} 4110 \mathrm{bc} 427$ ea $6 \mathrm{~b} 593 \mathrm{af} 4 \mathrm{fc} 27 \mathrm{fa0} c 1 \mathrm{a} 04124877742 \mathrm{e} 8224 \mathrm{~d} 3 \mathrm{aaabb} 2 \mathrm{~d} 0 \mathrm{e} 5932$ a3de0bc0239e483d249f484b40b40880a 4bdfd359c019f17ec980e0e138817fc932db08600 9fe26535fc84 860e301f853af41bc81af971c02e74d9e51293173739af83f8aa307fed16fb45ca08ef497a9fal61c4ecb2 $215 \mathrm{c} 297626 \mathrm{e} 4 \mathrm{cdb} 122877 \mathrm{dfc} 178554389118 \mathrm{e} 6925892 \mathrm{f} 4 \mathrm{e} 02 \mathrm{f} 3 \mathrm{da} 61449 \mathrm{f} 1873 \mathrm{~b} 8 \mathrm{e} 15 \mathrm{a} 7 \mathrm{f} 22465008 \mathrm{e} 647196$ b8d1c402e 78c4c505aaffa25c4a80bbc448d9d52072414eb6eb066bc9444ae3aed7a26f 49 dccc 19 ef 34948

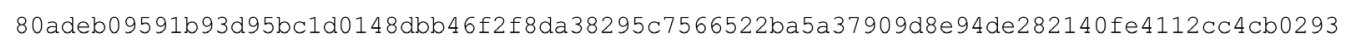

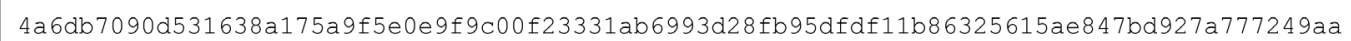
$6065 \mathrm{f} 64 \mathrm{cb} 68 \mathrm{~d} 1 \mathrm{de} 1018 \mathrm{~b} 40 \mathrm{a} 217 \mathrm{~b} 3 \mathrm{~d} 33 \mathrm{ad} 4 \mathrm{c} 65 \mathrm{ac} 74360 \mathrm{a} 8 \mathrm{~b} 5 \mathrm{fc} 7 \mathrm{~d} 39 \mathrm{fa} 32 \mathrm{~d} 6 \mathrm{a} 44 \mathrm{ecc} 103 \mathrm{~d} 6 \mathrm{be} 023 \mathrm{~b} 07 \mathrm{c} 9 \mathrm{c} 3660$ $85366829 \mathrm{~b} 66 \mathrm{fa} 19 \mathrm{~cd} 2 \mathrm{~d} 7 \mathrm{f} 448 \mathrm{f} 7623 f 75 \mathrm{~b} 454 \mathrm{a} 0 \mathrm{f} 03 \mathrm{~b} 1 \mathrm{df} 79 \mathrm{~b} 397 \mathrm{dc} 0644 \mathrm{be} 63424 \mathrm{baf} 75 \mathrm{dab} 09 \mathrm{a} 432 \mathrm{f} 6707833$ $68 \mathrm{e} 782 \mathrm{a} 1 \mathrm{cc} 4 \mathrm{bc} 6542 \mathrm{~b} 0 \mathrm{f} 8 \mathrm{df} 1 \mathrm{c} 73 \mathrm{a} 74 \mathrm{ef} 32 \mathrm{~d} 44 \mathrm{f} 6 \mathrm{~b} 4 \mathrm{~b} 0756826 \mathrm{da} 1 \mathrm{a} 93 \mathrm{a} 361 \mathrm{fb} 6 \mathrm{ee} 8 \mathrm{~d} 5181 \mathrm{cb} 15 \mathrm{f} 2 \mathrm{~d} 7$

$\mathrm{J}=16, i \mathrm{dx}=16$, type $=0, \operatorname{Pre16}=$

10000000100000000053484132353600000000000000000000000000000000000000000000000000000000 000000000000000000000000000000000000000000

IV16 =

Presented as 8 integers:

0xd60422aa 0x2c91c9e5 0xf0e28121 0xeda94d92 0xf24c4a68 0xbf8ab0b9 0x4d118432 0x8354983d Presented as a string of bytes:

aa2204d6e5c9912c2181e2f0924da9ed684a4cf2b9b08abf3284114d3d985483

The output digests, $\mathrm{H}=$

Presented as 8 integers:

0xf984dec6 0x48df8956 0x50f32833 0x638b076b 0xe4c18b61 0x887a9f35 0xa9ee17d3 0x6668d586 Presented as a string of bytes:

c6de84f95689df483328f3506b078b63618bcle4359f7a88d317eea986d56866

Figure 12. Test vector for SHA-256 16-lanes. 
Scientific Research Publishing (SCIRP) is one of the largest Open Access journal publishers. It is currently publishing more than 200 open access, online, peer-reviewed journals covering a wide range of academic disciplines. SCIRP serves the worldwide academic communities and contributes to the progress and application of science with its publication.

Other selected journals from SCIRP are listed as below. Submit your manuscript to us via either submit@scirp.org or Online Submission Portal.
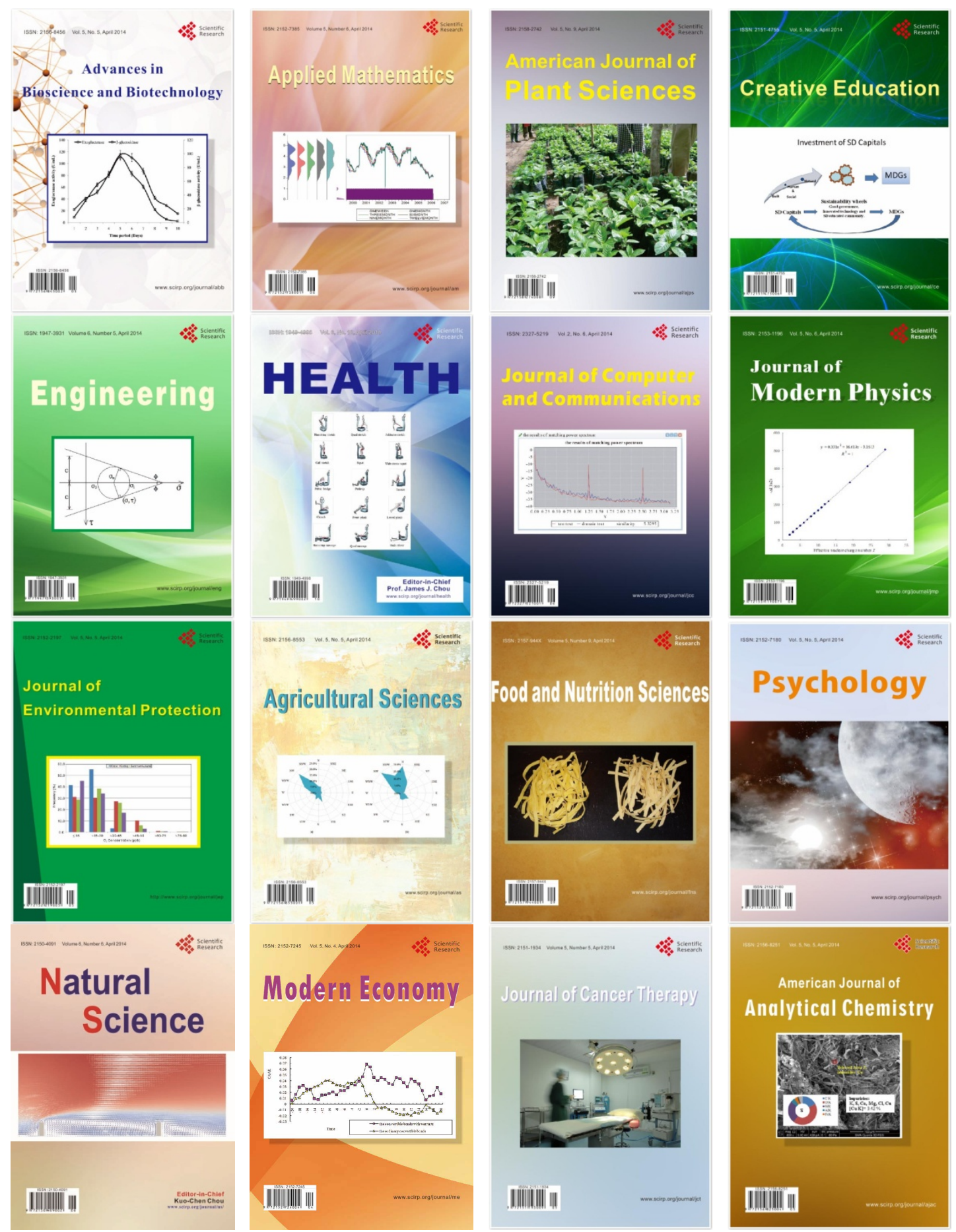